\title{
Correction to: Psychometric Evaluation of a Barriers to Mental Health Treatment Questionnaire for Latina/o/x Caregivers of Children and Adolescents
}

\author{
Alejandro L. Vázquez ${ }^{1}$ (D) Demi Culianos ${ }^{1}$ (D) . Cynthia M. Navarro Flores ${ }^{1}$ (D) \\ María de la Caridad Alvarez ${ }^{1}$ (D) . Tyson S. Barrett ${ }^{1}$ (D) . \\ Melanie M. Domenech Rodríguez ${ }^{1}$
}

Published online: 28 January 2022

(c) Springer Science+Business Media, LLC, part of Springer Nature 2022

\section{Correction to: Child \& Youth Care Forum https://doi.org/10.1007/s10566-021-09656-8}

While conducting analyses for a follow up study, the authors found errors in the interpretation of correlations reported in the "Utilization Outcomes" section of the results. The mental health service variables were negatively correlated with Barriers to Treatment Questionnaire-Latina/o/x Caregivers (BTQ-LC) scales because the outcomes were accidently coded incorrectly (i.e., yes [1] or no [2]). Mental health service utilization variables should have been coded with yes being associated with an increase in the chance that service utilization would occur (i.e., yes [1] or $n o[0])$. While the coefficients and significance of relationships (i.e., $p$ values) reported within the "Utilization Outcomes" section are unchanged, after correcting this coding error, the valence of the relationship changed with greater barriers on BTQ-LC scales being positively associated with the utilization of all mental health services examined. These findings likely reflect the nonclinical nature of the sample, as the majority of families reported experiencing few barriers to care and/or not needing mental health services as they were not clinically warranted. As a result, families who needed and utilized mental health services may have been more likely to encounter barriers to care. Despite this error, the primary analyses still suggest that the BTQ-LC represents a method of quantifying and studying barriers to youth mental health services among Latinx youth. The reported correlation coefficients and statistical significance values are accurate. The direction of the relationships were inverted such that positive relationships were reported as negative. The authors apologize for this error.

Publisher's Note Springer Nature remains neutral with regard to jurisdictional claims in published maps and institutional affiliations.

The original article can be found online at https://doi.org/10.1007/s10566-021-09656-8.

Alejandro L. Vázquez

Alejandro.Vazquez@aggiemail.usu.edu

1 Department of Psychology, Utah State University, 2810 Old Main Hill, 84322 Logan, UT, USA 